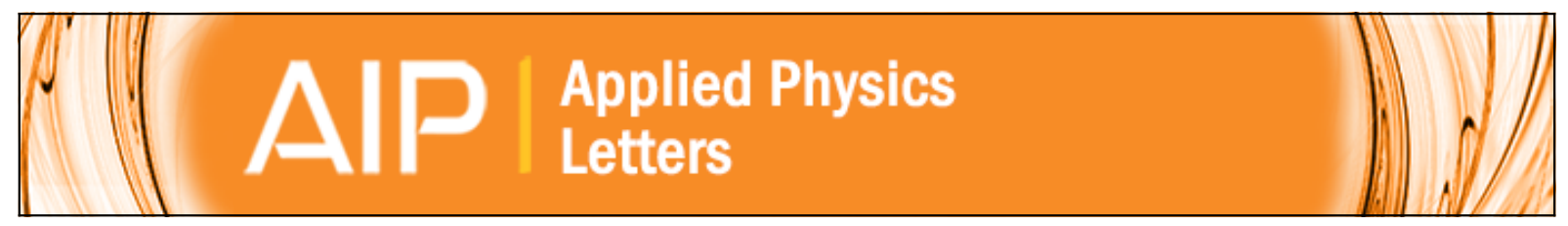

\title{
Resonant interband tunneling spin filter
}

David Z.-Y. Ting and Xavier Cartoixà

Citation: Applied Physics Letters 81, 4198 (2002); doi: 10.1063/1.1524700

View online: http://dx.doi.org/10.1063/1.1524700

View Table of Contents: http://scitation.aip.org/content/aip/journal/apl/81/22?ver=pdfcov

Published by the AIP Publishing

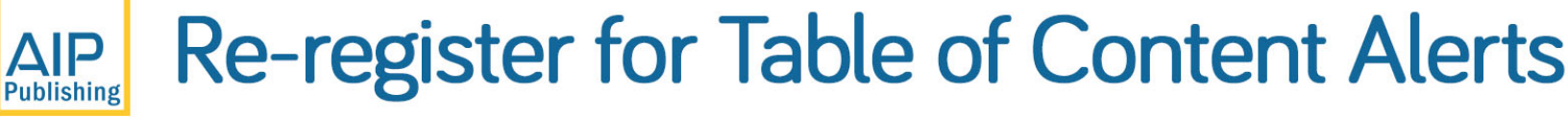

\section{Create a profile. \\ Sign up today!}




\title{
Resonant interband tunneling spin filter
}

\author{
David Z.-Y. Ting ${ }^{\text {a) }}$ \\ Jet Propulsion Laboratory, California Institute of Technology, Pasadena, California 91109
}

Xavier Cartoixà

T. J. Watson, Sr., Laboratories of Applied Physics, California Institute of Technology,

Pasadena, California 91125

(Received 23 July 2002; accepted 7 October 2002)

\begin{abstract}
We propose an InAs/GaSb/AlSb-based asymmetric resonant interband tunneling diode as a spin filter. The interband design exploits large valence band spin-orbit interaction to provide strong spin selectivity, without suffering from fast hole spin relaxation. Spin filtering efficiency is also enhanced by the reduction of tunneling through quasibound states near the zone center, where spin spitting vanishes and spin selectivity is difficult. Our calculations show that, when coupled with an emitter or collector capable of lateral momentum selectivity, the asymmetric resonant interband tunneling diode can achieve significant spin filtering in conventional nonmagnetic semiconductor heterostructures under zero magnetic field. () 2002 American Institute of Physics.
\end{abstract}

[DOI: $10.1063 / 1.1524700]$

A major research area in spintronics (spin-based electronics) is the development of efficient spin polarized current sources. ${ }^{1}$ An interesting candidate is the Rashba effect resonant tunneling spin filter made of nonmagnetic semiconductor heterostructures. ${ }^{2}$ The device uses structural inversion asymmetry (SIA) to create spin splitting, ${ }^{3}$ and exploits the fact that the spin of a resonantly transmitted electron aligns with that of the quasibound state traversed ${ }^{4,5}$ to achieve spin filtering. As illustrated in Fig. 1 of Ref. 6, spin directions of quantum well states are perpendicular to the growth direction ( $z$ axis) and to the in-plane wave vector $\mathbf{k}_{\|}{ }^{7}$ and, when plotted along with the constant energy contours of two spinsplit subbands in the $\mathbf{k}_{\|}$plane, appear as counterclockwise (CCW) and clockwise (CW) pinwheels [see also Fig. 4(b)]. This poses major challenges for the resonant tunneling spin filter. Within a given spin-split subband, which we label as $\mathrm{CCW}$ or $\mathrm{CW}$, states opposite in $\mathbf{k}_{\|}$have opposite spins. Thus, the ensemble of electrons resonantly transmitted through a given subband yields no net spin polarization. To solve this problem, Voskoboynikov and co-workers ${ }^{2}$ proposed the use of a small lateral (perpendicular to the growth direction) $E$-field in the source region to create an anisotropic lateral momentum distribution. ${ }^{2}$ Also, at a given $\mathbf{k}_{\|}$, states in the two spin-split subbands have opposite spins. While we exploit this property for spin filtering, we also require adequate resolution between the spin-split states. But this can be difficult, since the Rashba spin splitting is linearly proportional to $k_{\|}$near (and vanishes at) the zone center. ${ }^{3}$

To improve spin filtering efficiency, Koga and coworkers recently introduced a spin-blockade mechanism to provide much stronger discrimination between the $\mathrm{CCW}$ and $\mathrm{CW}$ current components. ${ }^{8}$ In this work we propose the resonant interband tunneling ${ }^{9}$ spin filter, which demonstrates large disparity between the transmission probabilities associated with the $\mathrm{CCW}$ and $\mathrm{CW}$ subbands, and significant reduction in zone center contributions.
Our device structure consists of an asymmetric composite InAs-GaSb well, surrounded by AlSb barriers and InAs electrodes. With appropriate layer widths, it can operate in either, under moderate bias, the intraband resonant tunneling regime [Fig. 1(a)] in which electrons traverse conduction subband states in InAs, or, under low bias, the resonant interband tunneling ${ }^{9}$ regime [Fig. 1(b)] where electrons traverse valence subband states in GaSb. In the interband design we exploit the strong spin-orbit interaction in valence bands for enhanced spin filtering, but collect electrons in the InAs conduction band to avoid fast spin relaxation in the valence bands. ${ }^{10,11}$

Figure 2 shows spin-dependent transmission coefficient spectra calculated within the effective bond orbital model ${ }^{12}$ using the multiband quantum transmitting boundary method. ${ }^{13}$ We intentionally align the spin axis of the incident electrons with that of the resonances probed ( $y$ axis, here). Figure 2(a) shows that in the intraband tunneling case, the resonant transmission probabilities through the two spin-split $n=1$ conduction subband states (cb1) are approximately equal. Figure 2(b) shows that in the interband tunneling case, transmission peak strength (height times width) through the highest heavy hole (hh1) states is much larger $(\approx 16$ times) for the $+y$ than the $-y$ spin polarization. This pronounced difference is a key property that we use to improve spinfiltering efficiency. (a) Intraband Tunneling

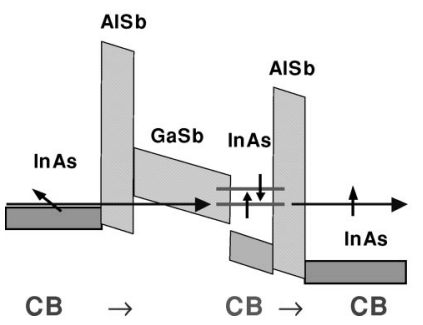

(b) Interband Tunneling

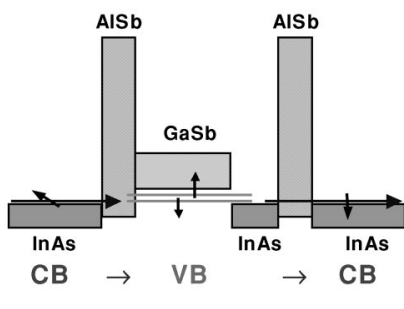

FIG. 1. Schematic energy band diagrams of the resonant intraband and interband tunneling spin filters. 


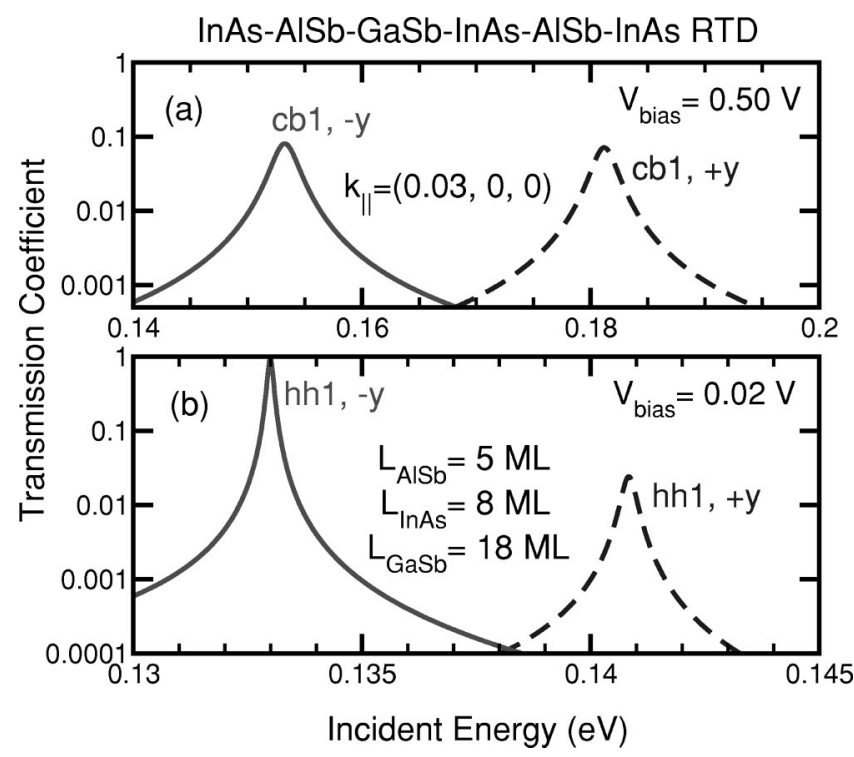

FIG. 2. Transmission coefficients of an asymmetric resonant tunneling diode operating in (a) the intraband and (b) the interband modes.

We have confirmed the same strong spin dependence in a $\mathbf{k} \cdot \mathbf{p}$ calculation. To understand its origin, we examine the properties of hh1 transmission resonances in Fig. 3. As spinorbit interaction increases with $k_{\|}$, both the $T_{\max }$ (peak height) and $\Delta E$ (peak width) differences between the $+y$ and the $-y$ spin channels also increase. However, the large difference in $T_{\max }$ is the primary source for the spindependence in peak strengths. A possible physical explanation of this result is provided by the analysis of double barrier resonant tunneling structures by Ricco and Azbel, ${ }^{14}$
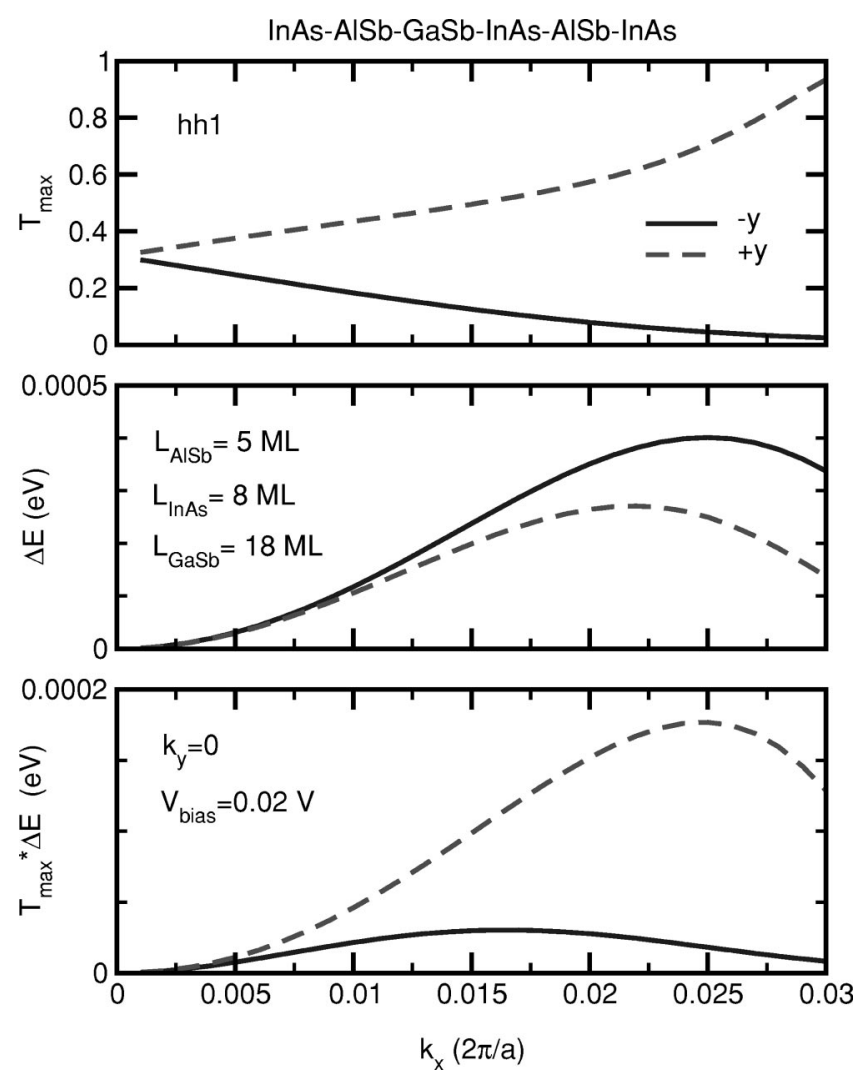

FIG. 3. Transmission resonance properties as functions of $\boldsymbol{k}_{\mathbb{1}}$ in a resonant interband tunneling spin filter.

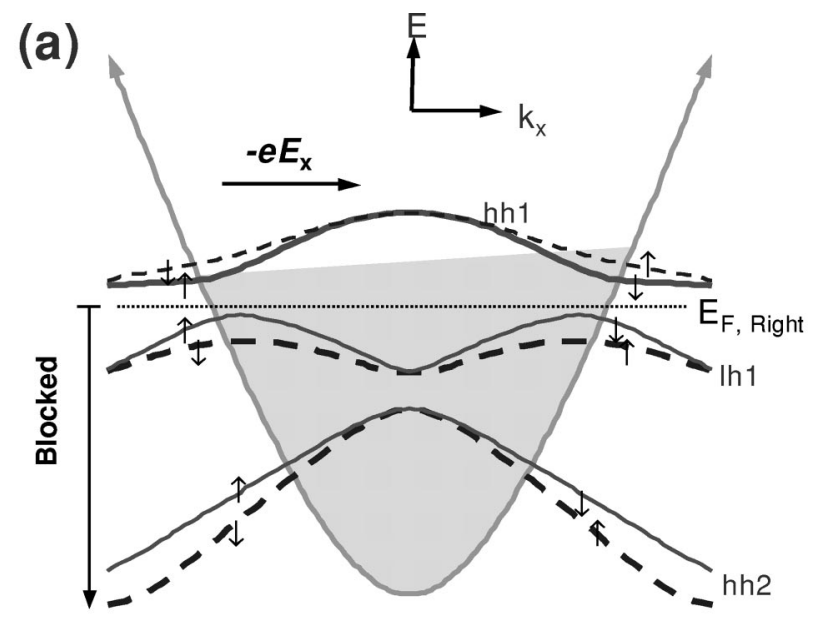

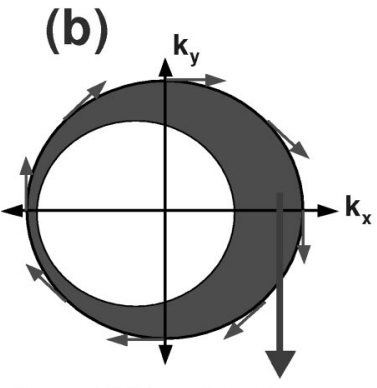

lower hh1 band (cw)

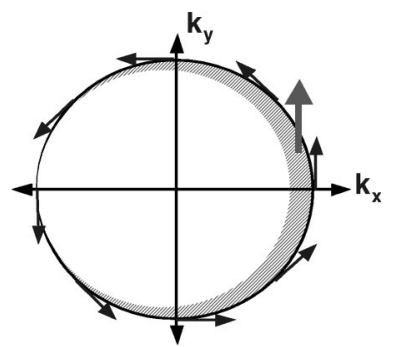

upper hh1 band (ccw)
FIG. 4. Schematic illustration of the resonant interband tunneling spin filter in, (a) energy-momentum space, and (b) momentum space.

which states that the peak transmission coefficient $T_{\max }$ is given by the ratio between the transmission probability of the strong barrier and that of the weak barrier. $T_{\max }=1$ is achieved only in symmetric structures, in which left and right barrier have equal strengths. In our case, the GaSb valance band quantum well is confined to the left by the AlSb barrier, and to the right by the combination of the (effectively thicker) InAs-AlSb barrier. Apparently, for the $-y$ spin, spin-orbit interaction strengthens the left barrier and weakens the right (recall that spin-orbit interaction is proportional to $\nabla V$, which has opposite signs at the two quantum well interfaces), effectively making the double barrier structure more symmetric, and thereby increasing $T_{\max }$. The opposite is true for the $+y$ spin. Thus as spin-orbit interaction increases with $k_{x}$, the difference between the transmission peak strengths of the two spin channels also increases.

Figure 3 shows that near the zone center, hh1 peak strengths are exceedingly weak due to the narrow hh1 resonance peak widths. This reduces contributions due to resonant tunneling through quasibound states near the zone center, where spins are difficult to resolve. Away from the zone center, the transmission resonance width $\Delta E$ increases rapidly due to hole mixing. ${ }^{15}$ The calculated tunneling escape times $(\tau=\hbar / \Delta E)$ for $k_{x}>0.0075(2 \pi / a)$ are shorter than 10 fs. The fast resonant tunneling process allows us to avoid significant spin relaxation while electrons transit the GaSb valence subbands.

Figure 4 illustrates the operating principles of the interband resonant tunneling spin filter. Figure 4(a) shows the valence subband structure of the spin-split quasibound states, together with the conduction band structure for the bulk InAs 

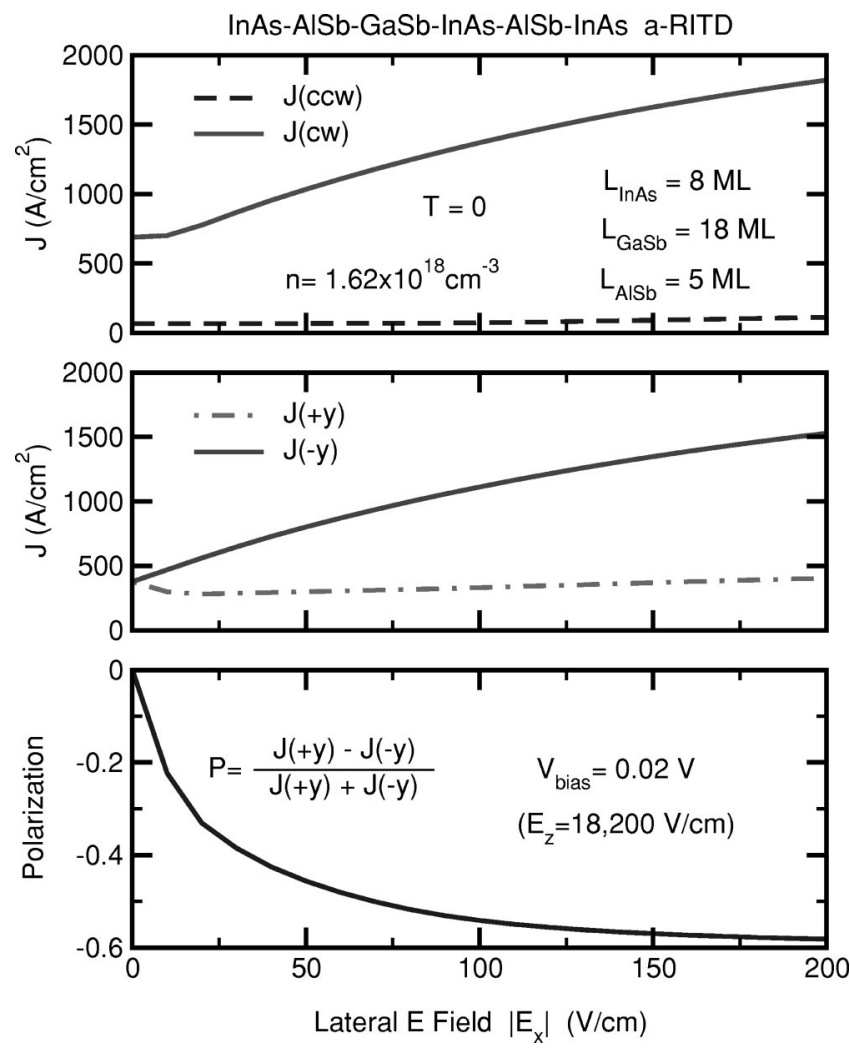

FIG. 5. Spin resolved current density components and spin polarization as functions of lateral $E$-field. A relaxation time of $1 \mathrm{ps}$ is used in the calculation.

emitter. The shaded region represents the emitter states available for resonant tunneling. The tilt in the top of the shaded region is the result of modification to the emitter Fermi level due to the applied lateral $E$-field, as described by the relaxation time approximation. ${ }^{16}$ We use the same doping level in the emitter and in the collector. The dotted line represents the collector Fermi level. At a bias of $0.02 \mathrm{~V}$, the occupied states in the collector block the light hole (lh1) and hh2 states, so that resonant interband tunneling takes place only through the hh1 states. Note that by setting the incident reservoir Fermi level below the hh1 band edge, we have another method of removing zone center contributions.

In Fig. 4(a), the overlap between the hh1 quasibound states and the available emitter states fulfills energy and inplane momentum conservation conditions for resonant tunneling. Its $\mathbf{k}_{\|}$-space representation is shown as the shaded areas in Fig. 4(b). The lateral $E$-field results in opposite net spins in the resonantly transmitted currents for each of the two spin-split hh1 subbands. However, strong spin- dependent tunneling allows the lower hh1 subband to dominate, yielding net spin in the total transmitted current.

Figure 5 shows the computed current densities and current polarizations as functions of lateral $E$-field for a resonant interband tunneling spin filter operating at a fixed transverse biasing voltage. Note that the lateral $E$-field is much smaller than the transverse $E$-field. Figure 5(a) shows the contributions from each of the spin-split subband bands separately. As a result of spin-dependent tunneling, $J_{C W}$ is more than an order of magnitude larger than $J_{C C W}$. In Fig. 5(b) we analyze the spins of transmitted electrons along the $y$ axis, and record separately current densities for spin up and spin down as $J_{+y}$ and $J_{-y}$, respectively. Figure 5(c) shows the current polarization $P$. For a modest value of $E_{x}$ $=70 \mathrm{~V} / \mathrm{cm}$, we can obtain $|P|>50 \%$. A similar device structure, highly optimized for intraband operation, yields $\approx 7 \%$. These theoretical results demonstrate that the interband tunneling concept can be used to improve the efficiency of the resonant tunneling spin filter ${ }^{2}$ significantly.

The authors thank D. L. Smith, D. H. Chow, J. S. Moon, J. N. Schulman, and T. C. McGill for helpful discussions. This work was sponsored by the Defense Advanced Research Projects Agency. A part of this work was carried out at the Jet Propulsion Laboratory, California Institute of Technology, through an agreement with NASA.

${ }^{1}$ S. A. Wolf, D. D. Awschalom, R. A. Buhrman, J. M. Daughton, S. von Molnar, M. L. Roukes, A. Y. Chtchelkanova, and D. M. Treger, Science 294, 1488 (2001).

${ }^{2}$ A. Voskoboynikov, S. S. Lin, C. P. Lee, and O. Tretyak, J. Appl. Phys. 87, 387 (2000).

${ }^{3}$ Y. A. Bychkov and E. I. Rashba, J. Phys. C 17, 6039 (1984).

${ }^{4}$ A. Voskoboynikov, S. S. Liu, and C. P. Lee, Phys. Rev. B 59, 12514 (1999).

${ }^{5}$ E. A. de Andrada e Silva and G. C. La Rocca, Phys. Rev. B 59, 15583 (1999).

${ }^{6}$ Th. Schäpers, G. Engels, J. Lange, Th. Klocke, M. Hollfelderm, and H. Lüth, J. Appl. Phys. 83, 4324 (1998).

${ }^{7}$ Neglecting anisotropy and microscopic bulk inversion asymmetry effects.

${ }^{8}$ T. Koga, J. Nitta, H. Takayanagi, and S. Datta, Phys. Rev. Lett. 88, 126601 (2002).

${ }^{9}$ J. R. Söderström, D. H. Chow, and T. C. McGill, Appl. Phys. Lett. 55, 1094 (1989).

${ }^{10}$ G. Fishman and G. Lampel, Phys. Rev. B 16, 820 (1977).

${ }^{11}$ T. C. Damen, L. Viña, J. E. Cunningham, J. Shah, and L. J. Sham, Phys. Rev. Lett. 67, 3432 (1991).

${ }^{12}$ Y. C. Chang, Phys. Rev. B 37, 8215 (1988); G. T. Einevoll and Y. C. Chang, Phys. Rev. B 40, 9683 (1989).

${ }^{13}$ D. Z.-Y. Ting, E. T. Yu, and T. C. McGill, Phys. Rev. B 45, 3583 (1992).

${ }^{14}$ B. Ricco and M. Ya. Azbel, Phys. Rev. B 29, 1970 (1984).

${ }^{15}$ D. Z.-Y. Ting, E. T. Yu, and T. C. McGill, Phys. Rev. B 45, 3576 (1992).

${ }^{16}$ See, for example, C. Kittel, Quantum Theory of Solids, 2nd rev. printing (Wiley, New York, 1987), pp. 179-184. 